\title{
DHHC8-Dependent PICK1 Palmitoylation is Required for Induction of Cerebellar Long-Term Synaptic Depression
}

\author{
Gareth M. Thomas, ${ }^{1,2,3}$ Takashi Hayashi, ${ }^{1,2,4}$ Richard L. Huganir, ${ }^{1,2}$ and David J. Linden ${ }^{2}$ \\ ${ }^{1}$ Howard Hughes Medical Institute and ${ }^{2}$ Department of Neuroscience, Johns Hopkins University School of Medicine, Baltimore, Maryland 21205, ${ }^{3}$ Shriners \\ Hospitals Pediatric Research Center and Department of Anatomy and Cell Biology, Temple University School of Medicine, Philadelphia, Pennsylvania \\ 19140, and ${ }^{4}$ Department of Molecular Neurobiology and Pharmacology, Graduate School of Medicine, The University of Tokyo, Bunkyo-ku, Tokyo 113- \\ 0033, Japan
}

The palmitoyl acyltransferase (PAT) DHHC8 is implicated in synaptic regulation but few DHHC8 substrates are known. Here we report that DHHC8 binds and palmitoylates the PDZ domain-containing protein PICK1 at a cysteine residue that is essential for long-term synaptic depression (LTD) in cultured mouse cerebellar Purkinje neurons. Cerebellar LTD is palmitoylation-dependent and induction of LTD requires DHHC8. Furthermore, PICK1 is a critical DHHC8 substrate whose palmitoylation is necessary for LTD. These results identify the first DHHC8 substrate required for a specific form of synaptic plasticity and provide new insights into synaptic roles of palmitoylation.

\section{Introduction}

AMPA-type glutamate receptor (GluA) targeting and trafficking is central to synaptic transmission and plasticity. GluA trafficking involves insertion and internalization via specific vesicles, and also lateral movement between different plasma membrane regions (Groc and Choquet, 2006; Shepherd and Huganir, 2007). Several direct binding partners regulate GluA trafficking. In particular, the PSD-95/discs large/ ZO-1 (PDZ) domain proteins glutamate receptor interacting protein-1 and -2 (GRIP1, GRIP2) and protein interacting with C-kinase (PICK1) bind and regulate GluA2-containing AMPARs (Shepherd and Huganir, 2007). However, it is not fully understood how PICK1 and GRIPs, which are predicted to be soluble cytosolic proteins, are targeted to the lipid-rich trafficking vesicles and specific membranes in which GluAs reside.

Cytosolic proteins can be targeted to specific membranes by direct lipid attachment. One protein-lipid attachment mechanism, palmitoylation, is emerging as critical for neuronal protein targeting and trafficking (Fukata and Fukata, 2010). However, despite palmitoylation's importance in neuronal regulation, few substrates of individual palmitoyl acyltransferases (PATs) are

Received March 21, 2013; revised July 24, 2013; accepted Aug. 14, 2013.

Author contributions: G.M.T., T.H., R.L.H., and D.J.L. designed research; G.M.T., T.H., and D.J.L. performed research; G.M.T., T.H., and D.J.L. analyzed data; G.M.T., T.H., R.L.H., and D.J.L. wrote the paper.

We thank Yilin Yu and Devorah VanNess for mouse cultures, Lisa Hamm for expert technical assistance, and DrY. Igarashi (Hokkaido University, Japan) for DHHC8 CDNA. This work was supported by funding from NHH and Howard Hughes Medical Institute (R.L.H.), NIH (D.J.L.), and Shriners Hospitals for Children (G.M.T.).

Under a licensing agreement between Millipore Corporation and The Johns Hopkins University, R.L.H. is entitled to a share of royalties received by the University on sales of products described in this article. R.L.H. is a paid consultant to Millipore Corporation. The terms of this arrangement are being managed by The Johns Hopkins University in accordance with its conflict-of-interest policies.

Correspondence should be addressed to either Richard L. Huganir or David J. Linden, Department of Neuroscience, Johns Hopkins University School of Medicine, 725 N Wolfe Street, Baltimore, MD 21205. E-mail: rhuganir@jhmi.edu or dlinden@jhmi.edu.

DOI:10.1523/JNEUROSCI.1283-13.2013

Copyright $\odot 2013$ the authors $\quad 0270-6474 / 13 / 3315401-07 \$ 15.00 / 0$ known. We have focused upon substrates of DHHC8, a PAT that is detected biochemically and immunocytochemically at and near synapses (Mukai et al., 2004; Trinidad et al., 2008; Thomas et al., 2012), in contrast to the ER/Golgi localization of many PATs (Ohno et al., 2006). Its synaptic localization suggests that DHHC8 may palmitoylate proteins that regulate synaptic transmission and plasticity, a hypothesis supported by the reduced hippocampal synapse number in DHHC8 knock-out mice ( $\mathrm{Mu}-$ kai et al., 2008). However, despite its likely importance for synaptic regulation, few DHHC8 substrates are known.

DHHC8 possesses a C-terminal PDZ ligand (Fukata and Fukata, 2010), which we hypothesized might allow DHHC8 to bind and subsequently palmitoylate PDZ domain proteins, perhaps including regulators of GluA trafficking. Here we identify the PDZ domain protein PICK1 as a DHHC8 interactor and substrate. PICK1 is essential for cerebellar LTD induction (Steinberg et al., 2006) and we report that cerebellar LTD induction is palmitoylation-dependent and requires both DHHC8 and PICK1-C414, the major PICK1 palmitoylation site in transfected cells. These requirements are likely linked because constitutively membrane-targeted PICK1 rescues cerebellar LTD deficits seen following DHHC8 knockdown. Together, these findings reveal a previously unappreciated role for DHHC8-dependent palmitoylation in cerebellar LTD and identify palmitoyl-PICK1 as critical for the LTD induction process.

\section{Materials and Methods}

Yeast two-hybrid screening and mammalian cell binding studies using the DHHC8 C terminus were performed as described previously (Thomas et al., 2012). Briefly, the DHHC8 "bait" and C-terminal 15 aa (Fig. 1A) were subcloned into the GST expression vector pCIS. The DHHC8 PDZ ligand was deleted by introducing a premature stop codon, using QuikChange mutagenesis. The same mutation was introduced into full-length human His-myc-DHHC8. Catalytically inactive DHHS8 was created by QuikChange. 
A

\section{DHHC8}

\section{'Bait'}
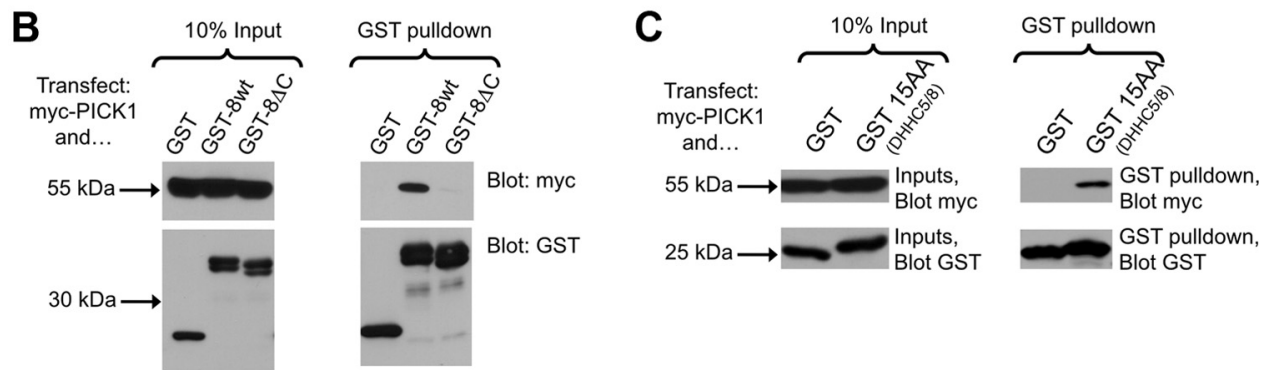

Figure 1. The DHHC8 C-terminal PDZ ligand binds PICK1. A, DHHC8 schematic, showing predicted transmembrane domains (blue) and catalytic DHHC domain (red). AC-terminal 15 aa sequence (yellow, identical in DHHC5) terminates in a PDZ ligand (ISV-C00, orange). The yeast two-hybrid bait region is indicated. B, HEK293T cells were transfected with myc-tagged full-length PICK1, plus either GST alone (GST), a GST fusion of the DHHC8 C terminus (GST-8wt), or a GST fusion of the DHHC8 C terminus lacking the PDZ ligand (GST-8 $\Delta$ C). Inputs (left) and GST pull-downs (right) were immunoblotted with the indicated antibodies. C, The DHHC5/8 common C terminus is sufficient to bind PICK1. HEK293T cells were cotransfected with myc-PICK1 plus either GST or a GST fusion of the DHHC5/8 C-terminal 15 aa (GST-15AA DHHC5/8). Inputs (left) and GST pull-downs (right) were immunoblotted with the indicated antibodies.

An shRNA (CTTCAGTATGGCTACCTTCAT, which perfectly matches both murine and rat DHHC8) effectively and specifically knocked down cotransfected DHHC8 in HEK293T cells. The same shRNA, subcloned into a lentiviral vector and used to infect cultured cortical neurons, knocked down endogenous DHHC8 (see supplemental data in Thomas et al., 2012). Human DHHC8 cDNA, which contains three mismatches in the shRNA target sequence, was used as a "rescue" cDNA for mouse neuron experiments. PICK1-C414S was generated by PCR using mutagenic primers. A minimal prenylation sequence from paralemmin, which does not affect dendritic spines or filopodia (Gauthier-Campbell et al., 2004), was added to the PICK1-C414S C terminus by PCR.

Radioactive ( ${ }^{3} \mathrm{H}$-palmitate labeling) and nonradioactive (acylbiotinyl exchange) palmitoylation assays were performed on cortical neurons, transfected cell lysates, or P21 mouse brain regions, as described previously (Thomas et al., 2012).

Preparation of cerebellar cultures from embryonic mice of either sex, lentiviral infection, biolistic transformation, and voltage-clamp recording were performed essentially as described previously (Takamiya et al., 2008). PICK1 knock-out was achieved by infecting PICK1 conditional knock-out (CKO) mouse cultures (Gardner et al., 2005) at DIV1 with lentivirus expressing Cre recombinase. PATs were knocked down by infecting neurons from wild-type (PICK1wt) or PICK1 CKO mice with lentiviruses expressing eGFP plus shRNAs, or eGFP alone. Purkinje neurons (PNs) were used at DIV12-DIV14. When different viruses were compared, the total viral load added to each culture was kept constant.

\section{Results}

A yeast two-hybrid screen performed using the DHHC8 C terminus (Fig. 1A) identified five clones that coded for the PDZ domain protein PICK1. To confirm this interaction in mammalian cells, we fused the DHHC8 bait to an N-terminal GST tag. GSTDHHC8 robustly bound PICK1 in cotransfected HEK293T cells (Fig. 1B). In contrast, neither GST alone nor GST-DHHC8 lacking the PDZ ligand, bound PICK1 (Fig. $1 B$ ), suggesting that the DHHC8 C terminus directly binds the PICK1 PDZ domain. Indeed, a GST fusion of the DHHC8 C-terminal 15 aa, which are identical in the closely related DHHC5, was sufficient to bind PICK1 (Fig. 1C). A GST-DHHC5 C-tail fusion also bound PICK1 in a PDZ ligand-dependent manner (data not shown).

We previously identified another DHHC5/8 interactor, GRIP1b, as a DHHC5/8 substrate (Thomas et al., 2012). To determine whether these PATs also palmitoylate PICK1, we transfected HEK293T cells with myc-PICK1 and performed a nonradioactive palmitoylation assay, acyl-biotin exchange (ABE), which specifically purifies palmitoylated proteins from cell lysates (Thomas et al., 2012). Myc-PICK1 was detected at low levels in ABE samples from HEK293T cells coexpressing empty vector, but was robustly detected from cells coexpressing DHHC8, suggesting that DHHC8 palmitoylates PICK1. Cys414, close to PICK1's C terminus, is a bioinformatically predicted palmitoylation site (Zhou et al., 2006) (Fig. 2A) and mutating Cys414 to nonpalmitoylatable Ser greatly reduced PICK1 palmitoylation by DHHC8, judged by the reduced level of PICK1C414S in ABE samples (Fig. 2B, quantified in Fig. 2C). This strongly suggests that DHHC8 palmitoylates PICK1-C414. DHHC5 also robustly palmitoylated PICK1-C414, as judged by ABE (data not shown).

We next addressed whether endogenous PICK1 is palmitoylated in brain and in cultured neurons. PICK1 was clearly detected in forebrain and cerebellar $\mathrm{ABE}$ fractions, indicative of palmitoylation (Fig. $2 D$ ). In $\left[{ }^{3} \mathrm{H}\right]$ palmitate-labeled cortical neurons, a PICK1 N-terminal antibody, but not nonspecific IgG, efficiently immunoprecipitated PICK1 (Fig. 2E). Autoradiography revealed a $\left[{ }^{3} \mathrm{H}\right]$-palmitate band only in PICK1 immunoprecipitates, which comigrated with PICK1. Interestingly, a PICK1 C-terminal antibody also immunoprecipitated PICK1, but these immunoprecipitates lacked a detectable $\left[{ }^{3} \mathrm{H}\right]$-palmitate signal. A plausible explanation for this finding is that PICK1 C-terminal palmitoylation prevents immunoprecipitation by the C-terminal antibody, suggesting that, as in heterologous cells, the major PICK1 palmitoylation site is Cys414. Both N- and C-terminal immunoprecipitates contain similar amounts of PICK1 (Fig. 2E), suggesting that only a small fraction of PICK1 is likely palmitoylated in neurons, consistent with $\mathrm{ABE}$ results.

Even if the total cellular complement of PICK1 is not highly palmitoylated, a specific palmitoyl-PICK1 pool may play essential roles. PICK1 controls cerebellar LTD by stabilizing endocytosed GluA2 AMPAR subunits intracellularly (Steinberg et al., 2006). Although palmitoylation is implicated in regulation of synaptic transmission (El-Husseini et al., 2002), it is unknown whether cerebellar LTD is palmitoylation-dependent. To address this possibility, we incubated cultured cerebellar neurons with the palmitoylation inhibitor 2-bromopalmitate (2-Br; Jennings et al., 2009; Fukata and Fukata, 2010) before LTD induction. 2-Br 
A

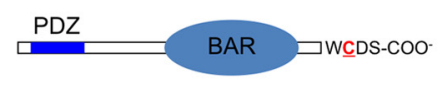

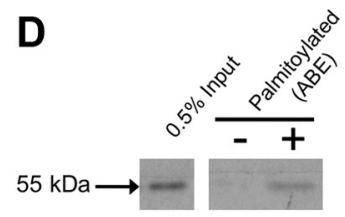

Forebrain

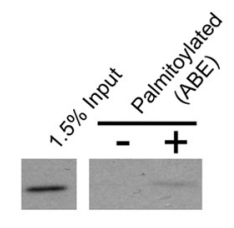

Cerebellum

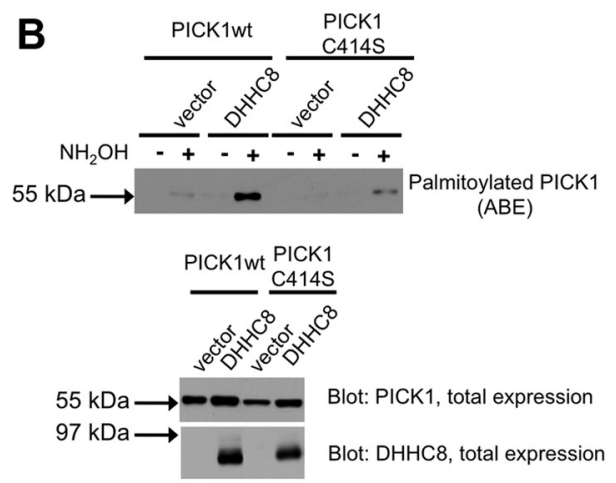

E
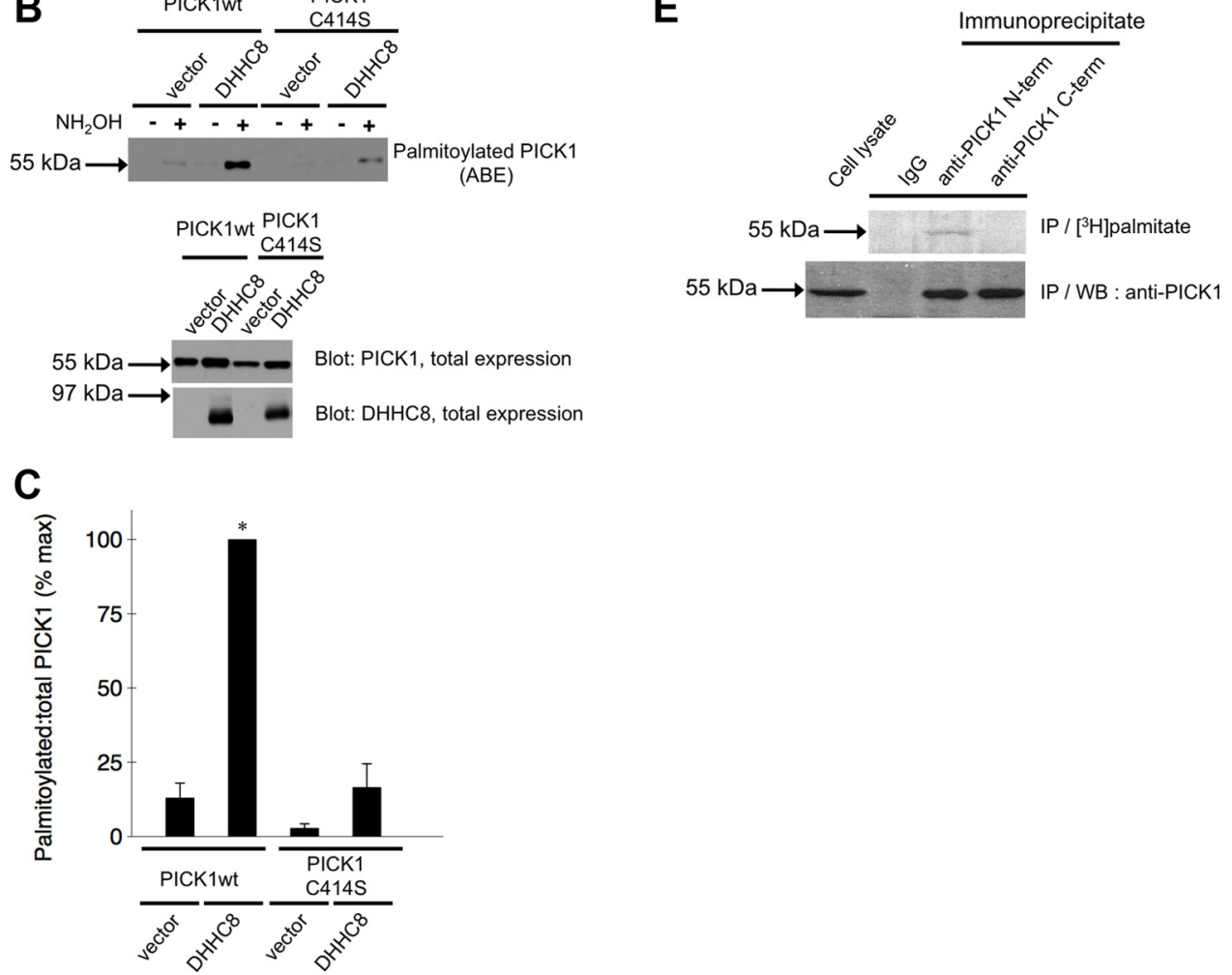

Figure 2. DHHC8 palmitoylates PICK1 at Cys414, and PICK1 is endogenously palmitoylated in neurons. A, PICK1 Schematic, showing PDZ and BAR domains, plus C-terminal residues critical for actin binding (W413) and palmitoylation (C414, red underlined). B, HEK293T cells were transfected with His-myc-DHHC8 and either wild-type or point mutant (C414S) myc-PICK1. Palmitoylproteins were isolated by ABE and PICK1 levels in ABE samples were detected by immunoblotting (top). Lysates were blotted to detect total PICK1 (middle) and His-myc-DHHC8 (bottom) levels. Note that palmitoyl-PICK1 isolation requires hydroxylamine $\left(\mathrm{NH}_{2} \mathrm{OH}\right)$, an essential ABE component. C, Immunoblots from multiple experiments as in Figure $2 B$ ( $\mathrm{PICK} 1 \mathrm{wt}, 6$ determinations per condition, PICK1-C414S, 3 determinations per condition) were quantified to detect palmitoylated and total PICK1 levels. Mean palmitoylated:total PICK1 signal is plotted as a percentage of maximum (PICK1wt + DHHC8). Error bars indicate SEM. ${ }^{*} p<0.05$ compared with PICK1 + vector alone, $t$ test. $\boldsymbol{D}$, Neuronal PICK1 is endogenously palmitoylated. The indicated juvenile rat brain regions were homogenized and subjected to ABE to purify palmitoyl proteins. ABE samples and homogenates were blotted to detect PICK1.E, Cultured cortical neurons metabolically labeled with [ $\left.{ }^{3} \mathrm{H}\right]$-palmitate were lysed and immunoprecipitated with the indicated antibodies. $\left[{ }^{3} \mathrm{H}\right]$ signal (top) and PICK1 protein levels (bottom) are shown.

(20 $\mu \mathrm{M}$, preincubated for $>30 \mathrm{~min}$ ) blocked cerebellar LTD almost completely (Fig. 3A).

We routinely induce and monitor LTD using glutamate test pulses, which allows analysis of postsynaptic mechanisms in isolation (Steinberg et al., 2006), but measures both synaptic and extrasynaptic receptors. To determine whether palmitoylation is required for synaptic AMPAR removal, we treated cultures with 2-Br as above, measured miniature EPSCs (mEPSCs), and then induced LTD chemically by bathapplying the PKC activator phorbol-12,13-diacetate (PDA; $200 \mathrm{nM}$ ). PDA produced an LTD-like effect, appearing as a slowly developing, but ultimately stable, decrease in mEPSC mean amplitude (Linden and Connor, 1991; Steinberg et al., 2006). This chemical form of cerebellar LTD generally followed results seen with glutamate/depolarization pairing, with 2-Br-pretreated cells showing no depression. This indicates that palmitoylation is required for depression of synaptic AMPA receptor currents and that blocking palmitoylation is unlikely to prevent LTD through effects on mGluR1 or voltage-sensitive calcium channels, which are required for pairing-induced but not PDA-evoked chemical LTD (data not shown).

This result suggests that palmitoylation is critical for cerebellar LTD but does not identify the key palmitoyl-protein(s) involved. To determine whether palmitoyl-PICK1 is critical for cerebellar LTD, PICK1 CKO cerebellar cultures were infected with lentivirus-expressing Cre recombinase. This approach minimizes the likelihood of developmental effects of knock-out of PICK1 and was used previously to delete other PDZ domain proteins in cerebellar cultures (Takamiya et al., 2008). Consistent with previous findings (Steinberg et al., 2006), cerebellar LTD was absent in PICK1 CKO PNs, but was rescued by transfecting PICK1wt cDNA. In contrast, no rescue was observed with PICK1-C414S cDNA (Fig. $3 A$ ). In parallel experiments, we measured chemical LTD of mEPSCs. Similar to conventional PICK1 KO PNs (Steinberg et al., 2006), PICK1 CKO PNs showed no depression. Transfecting PICK1wt, but not PICK1-C414S, rescued this phenotype (Fig. 3B). 



Figure 3. Cerebellar LTD in cultured PNs depends on palmitoylation and a specific PICK1 palmitoylation site. $\boldsymbol{A}$, Wild-type mouse cultures were treated for $>30$ min with the indicated concentrations of 2-bromopalmitate or with vehicle (EtOH, $<0.02 \%)$. After baseline recording of glutamate-evoked inward currents, LTD was induced by iontophoretic glutamate pulses in conjunction with depolarization. Six pairings were delivered at $t=0 \mathrm{~min}$ (horizontal bar) and 12 pairings at $t=22.5 \mathrm{~min}$ (horizontal bar, $2 \times$ ). Representative single current traces were acquired at the indicated times. Scale bars, 1 s, 40 pA. PICK1 CK0 mouse cultures were infected with Cre-expressing lentivirus at DIV1 and transfected with PICK1wt, PICK1-C414S, or PICK1-W413A CDNAs at DIV9-DIV10 before baseline recording and LTD induction at DIV13-DIV14. PICK1 ${ }^{-1-}$; PICK1wt plasmid $(n=6)$; PICK1 ${ }^{-1-}$; PICK1 CS mutant plasmid $(n=8)$; PICK1 ${ }^{-1-}$; PICK1 W413A actin-binding mutant plasmid ( $n=7)$; WT: 2-Br-palmitate, $10 \mu \mathrm{m}$, preincubated $(n=5)$; WT: 2-Br-palmitate (20 $\mu \mathrm{m}$, preincubated $(n=7)$; WT; vehicle $(n=6)$. Normalized current amplitude at $t=40$ min was compared using the Mann-Whitney $U$ test. This revealed a significant difference between the PICK $1^{-1-}$; PICK1 WT plasmid and PICK ${ }^{-l-}$; PICK1 CS mutant plasmid groups $(p<0.001)$ and between the WT; vehicle and WT, 2-Br-palmitate $20 \mu \mathrm{m}$ groups $(p<0.001)$. $\boldsymbol{B}$, For each condition in $\boldsymbol{A}$, we attempted to induce chemical LTD by bath applying the PKC activator PDA (200 nM) at $t=0-10 \mathrm{~min}$ as indicated by the horizontal bar. mEPSCs were measured and are expressed as mean amplitudes. $n=10$ cells/group. $\mathrm{mEPSC}$ amplitudes at $t=45 \mathrm{~min}$ were compared using the Mann-Whitney $U$ test. This revealed a significant difference between the PICK1 ${ }^{-1-}$; PICK1 WT plasmid and PICK1 ${ }^{-1-}$; PICK1 $\mathrm{CS}$ mutant plasmid groups $(p<0.02)$ and between the WT; vehicle and WT, 2-Br-palmitate $20 \mu$ m groups $(p<0.02)$. C, DHHC8 palmitoylates PICK1-W413A. HEK293T cells were transfected with His-myc-DHHC8 plus either wild-type or actin-binding mutant (W413A) myc-PICK1. ABE samples were blotted to detect palmitoyl-PICK1 (top). Lysates were blotted to detect PICK1 (middle) and His-myc-DHHC8 (bottom) total expression.

Inhibition of palmitoylation and mutation of PICK1-C414, the major PICK1 palmitoylation site in heterologous cells, similarly blocked cerebellar LTD. Together, these results suggest a requirement for C414 palmitoylation in cerebellar LTD. However, a neighboring residue (W413) reportedly controls PICK1dependent actin regulation (Rocca et al., 2008; Nakamura et al., 2011). C414 mutation could thus indirectly affect PICK1 function by altering this actin-regulating motif. However, a PICK1 mutant that cannot regulate actin (PICK1-W413A; Rocca et al., 2008) fully rescued cerebellar LTD in PICK1 CKO neurons, judged both by monitoring pairing-evoked LTD with glutamate test pulses and PDA-evoked chemical LTD of mEPSCs (Fig. $3 A, B)$. Moreover, DHHC8 palmitoylated PICK1-W413A and PICK1wt to a similar extent in heterologous cells (Fig. 3C). This suggests that the effects of PICK1-C414S mutation are independent of PICK1's role in actin regulation.

To test further possible links between PICK1 palmitoylation and cerebellar LTD, we addressed whether DHHC5 and DHHC8, which palmitoylate PICK1 in transfected cells and are two of only four PATs detected in PNs (Fang et al., 2006; Doyle et al., 2008) 

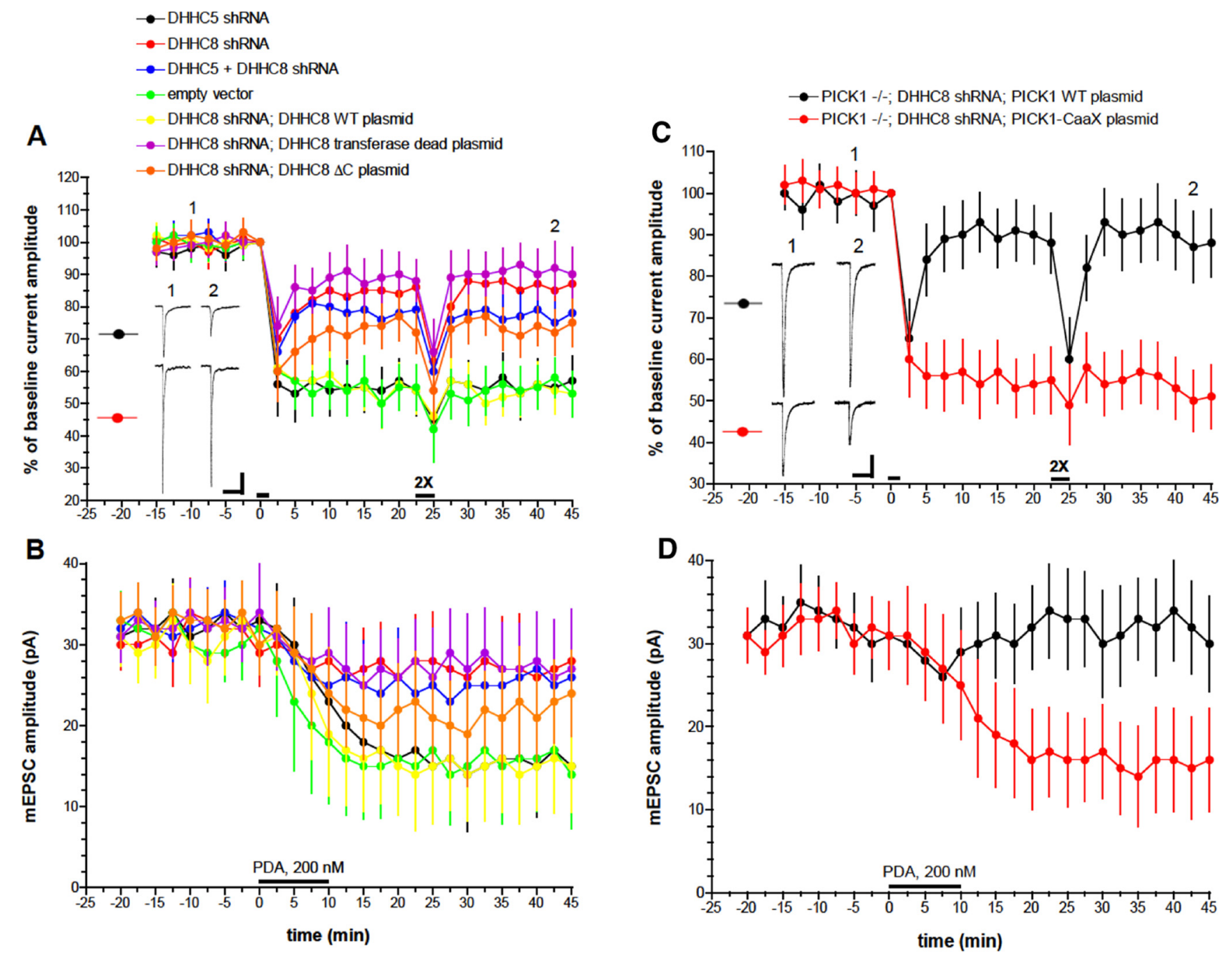

Figure 4. Cerebellar LTD in cultured PNs depends on DHHC8 catalytic activity, and palmitoyl-PICK1 is a key DHHC8 substrate that controls LTD. A, Wild-type mouse cultures were infected with lentiviruses expressing the indicated shRNAs. A subset of cultures infected with DHHC8 shRNA was subsequently transfected with the indicated DHHC8 rescue constructs. Baseline recording and LTD induction were performed as in Figure 3. Scale bars, 1 s, 50 pA. DHHC5 shRNA $(n=8)$; DHHC8 shRNA $(n=8)$; DHHC5 + DHHC8 shRNA $(n=7)$; empty vector $(n=7)$; DHHC8 shRNA; DHHC8 WT plasmid $(n=9)$; DHHC8 shRNA; DHHS8 transferase dead plasmid $(n=8)$; DHHC8 shRNA; DHHC8 $\Delta$ C plasmid $(n=7)$. Normalized current amplitude at $t=40$ min was compared using the Mann-Whitney $U$ test. This revealed a significant difference between the empty vector and DHHC8 shRNA groups $(p<0.001)$ and between the DHHC8 shRNA; DHHC8 WT plasmid and DHHC8 shRNA; DHHC8 transferase dead plasmid groups $(p<0.001)$. $\boldsymbol{B}$, For each condition in $A$, PDA (200 nm) was bath applied to induce chemical LTD and mEPSCs were measured. $N=10$ cells/group. $\mathrm{mEPSC}$ amplitudes at $t=45 \mathrm{~min}$ were compared using the Mann-Whitney $U$ test. This revealed a significant difference between the empty vector and DHHC 8 shRNA groups $(p<0.05)$ and between the DHHC8 shRNA; DHHC8 WT plasmid and DHHC8 shRNA; DHHS8 transferase dead plasmid groups $(p<0.05)$. C, PICK1 CKO mouse cultures were coinfected with two lentiviruses, one expressing Cre and the second expressing DHHC8 shRNA and subsequently transfected with either PICK1wt or the palmitoylation-mimicking PICK1-CaaX. Baseline recording and LTD induction were performed as in Figure 3 A. Scale bars, 1 s, 50 pA. PICK1 ${ }^{-1-}$; DHHC8 shRNA; PICK1 WT plasmid $(n=9) ;$ PICK1 $^{-1-}$; DHHC8 shRNA; PICK1-CaaX plasmid $(n=10)$. Normalized current amplitude at $t=40$ min was compared using the Mann-Whitney $U$ test. This revealed a significant difference between the two groups $(p<0.001)$. D, For each condition in C, PDA was bath applied to induce chemical LTD and $\mathrm{mEPSCS}$ were measured. $N=10$ cells/group. $\mathrm{mEPSC}$ amplitudes at $t=40 \mathrm{~min}$ were compared using the Mann-Whitney $U$ test. This revealed a significant difference between the two groups $(p<0.02)$.

are required for cerebellar LTD. We infected neurons from wildtype mice with lentiviruses expressing GFP alone, or GFP plus shRNAs to knock down DHHC5, DHHC8, or both PATs. We previously demonstrated the effectiveness and specificity of DHHC5 and DHHC8 shRNAs (Thomas et al., 2012). Strikingly, cerebellar LTD was unaffected in DHHC5-knockdown neurons, but was greatly reduced (although not eliminated) in DHHC8knockdown neurons (Fig. 4A). Cerebellar LTD in DHHC5 + DHHC8 knockdown neurons was slightly less affected than in DHHC8-knockdown neurons (Fig. 4A), a result likely due to lower DHHC8 shRNA viral expression in the DHHC5+DHHC8 condition, compared with DHHC8 shRNA virus alone. Again, PDA-evoked chemical LTD of mEPSCs mirrored results seen with glutamate/depolarization-induced pairing (Fig. 4B). These results suggest that DHHC8, but not DHHC5, is required for full expression of cerebellar LTD, although other PN PATs may be important in this process.

To address potential off-target shRNA effects, we attempted to rescue cerebellar LTD with shRNA-resistant DHHC8 constructs. Reassuringly, shRNA-resistant DHHC8 wild-type cDNA (DHHC8wt) fully rescued cerebellar LTD. Catalytically inactive DHHS8 did not rescue LTD, while catalytically active DHHC8 lacking the PDZ ligand (DHHC8 $\triangle \mathrm{C}$ ) partially rescued LTD (Fig. 4A). A similar pattern was observed for PDA-evoked chemical LTD of mEPSCs, which was fully rescued by DHHC $8 \mathrm{wt}$, not rescued by DHHS8, and incompletely rescued by DHHC8 $\Delta$ C (Fig. $4 A$ ). These results suggest that 
DHHC8 targets during LTD include PDZ domain proteins, although the partial rescue by $\mathrm{DHHC} 8 \mathrm{C}$ suggests either that the strict dependence of PDZ binding is partially overridden when DHHC8 $\Delta$ C is overexpressed and/or that DHHC8 also palmitoylates additional, non-PDZ domain substrates to control LTD.

The requirement for palmitoylation, DHHC8, and PICK1 for cerebellar LTD implicated PICK1 as a critical DHHC8 substrate that controls LTD. If so, mimicking constitutive PICK1 palmitoylation might compensate for DHHC8 knockdown. We therefore generated a constitutive lipid-attached PICK1, PICK1-CaaX, by adding a prenylation sequence to the PICK1-C414S C terminus i.e., close to the likely PICK1 palmitoylation site. This approach successfully mimics constitutive palmitoylation of another PDZ domain protein, PSD-95 (El-Husseini et al., 2002) and effectively targeted PICK1 to postsynaptic membranes in transfected neurons (data not shown). In PICK1 CKO neurons coinfected with Cre-expressing lentivirus to knock out PICK1 and with DHHC8 shRNA-expressing lentivirus, transfected PICK1wt could not rescue LTD, which was reduced to a similar level to that seen following DHHC8 knockdown (compare Fig. 4, $A$ and $C$ ). However, transfected PICK1-CaaX restored cerebellar LTD to wild-type levels. PDA-evoked chemical LTD revealed similar effects, with depression essentially absent following PICK1wt rescue, but restored to normal levels by PICK1-CaaX rescue (Fig. $4 D)$. Together, these findings suggest that PICK1 is indeed a critical DHHC8 substrate required for cerebellar LTD.

\section{Discussion}

Despite its emerging importance in synaptic regulation (Fukata and Fukata, 2010), roles of palmitoylation in synaptic plasticity remain largely unexplored. We report here that cerebellar LTD is palmitoylation-dependent and requires PICK1-C414, a major palmitoylation site in transfected cells. DHHC8, one of a limited subset of PN PATs, binds and palmitoylates PICK1 in heterologous cells. shRNA knockdown reveals DHHC8's importance for cerebellar LTD and rescue experiments suggest that PICK1 is the key DHHC8 substrate required for cerebellar LTD.

Cerebellar LTD requires two GluA2 interactors, PICK1 and GRIP1 (Steinberg et al., 2006; Takamiya et al., 2008). Both PICK1 and the GRIP1b isoform are palmitoylated (this report and Thomas et al., 2012). However, GRIP1b palmitoylation is dispensable for cerebellar LTD as GRIP1bwt and nonpalmitoylatable (C11S) GRIP1b both rescue cerebellar LTD in GRIP1/GRIP2 double knock-out (DKO) PNs (DJL, GMT, RLH: our unpublished observations). This is consistent with the rescue of cerebellar LTD in GRIP1/2 DKO neurons by the nonpalmitoylated GRIP1a isoform (Takamiya et al., 2008).

In contrast, PICK1-C414S cannot rescue LTD in PICK1 CKO neurons. C414 is the major PICK1 site palmitoylated by DHHC8, a PAT that is required for full expression of cerebellar LTD. This suggests that C414 mutation blocks cerebellar LTD by preventing PICK1 palmitoylation. However, C414 abuts W413, via which PICK1 binds Arp2/3 and regulates LTD-associated AMPAR removal and spine shrinkage in hippocampal neurons (Nakamura et al., 2011). Our data suggest that, at least in PNs, PICK1's different roles controlled by these neighboring residues are independent. Not only is PICK1's actin-binding ability dispensable for LTD, but cerebellar LTD occurs without changing PN spine size or number (Sdrulla and Linden, 2007). These findings reinforce the notion that different mechanisms underlie forebrain and cerebellar LTD. However, we cannot exclude the possibility that C414 mutation affects other roles of PICK1 independent of effects on palmitoylation.
How, might palmitoyl-PICK1 regulate GluA2 trafficking? PICK1's PDZ domain binds C-terminal ligands weakly (Madsen et al., 2005), so palmitoylation, perhaps together with other lipidbinding motifs (Jin et al., 2006), may enhance PICK1 membrane binding, thus stabilizing associations with transmembrane GluA2. Alternatively, PICK1 palmitoylation may increase PICK1-GluA2 targeting to membrane microdomains (Greaves et al., 2009) linked to receptor internalization.

DHHC8 knockdown incompletely blocks cerebellar LTD, suggesting that at least one other PAT(s) can palmitoylate PICK1. DHHC5, which also palmitoylates PICK1 in transfected cells, appears to be the best candidate, but DHHC5 knockdown does not affect cerebellar LTD alone and does not further reduce the magnitude of LTD when combined with DHHC8 knockdown (Fig. 4). Lack of compensation by DHHC5 is perhaps unsurprising, because in hippocampal neurons, DHHC5 localizes predominantly to dendritic shafts, while DHHC8 is more synaptic (Thomas et al., 2012). In addition to differential subcellular localization, protein expression level differences could also prevent DHHC5 from compensating for DHHC8. We note that structural and functional abnormalities in DHHC8 knock-out neurons (Mukai et al., 2008) provide a precedent for lack of compensation for DHHC8 by DHHC5.

Only two other PATs (DHHC3/GODZ and DHHC14) are reported in PNs (Fang et al., 2006; Doyle et al., 2008). Of these, the more likely to compensate for DHHC8 is DHHC3/GODZ, which also contains a PDZ ligand (Fukata and Fukata, 2010) and palmitoylates PICK1 in transfected cells (G.M.T., R.L.H., unpublished observations). These findings also imply that the limited number of PATs expressed by a specific cell type may counterbalance the overlapping PAT specificity sometimes reported in transfected cells (Fukata and Fukata, 2010).

Together, our data suggest that DHHC8 is a major PICK1 PAT in PNs and that palmitoylation is critical for PICK1's role in cerebellar LTD.

\section{References}

Doyle JP, Dougherty JD, Heiman M, Schmidt EF, Stevens TR, Ma G, Bupp S, Shrestha P, Shah RD, Doughty ML, Gong S, Greengard P, Heintz N (2008) Application of a translational profiling approach for the comparative analysis of CNS cell types. Cell 135:749-762. CrossRef Medline

El-Husseini Ael-D, Schnell E, Dakoji S, Sweeney N, Zhou Q, Prange O, Gauthier-Campbell C, Aguilera-Moreno A, Nicoll RA, Bredt DS (2002) Synaptic strength regulated by palmitate cycling on PSD-95. Cell 108: 849-863. CrossRef Medline

Fang C, Deng L, Keller CA, Fukata M, Fukata Y, Chen G, Lüscher B (2006) GODZ-mediated palmitoylation of GABA(A) receptors is required for normal assembly and function of GABAergic inhibitory synapses. J Neurosci 26:12758-12768. CrossRef Medline

Fukata Y, Fukata M (2010) Protein palmitoylation in neuronal development and synaptic plasticity. Nat Rev Neurosci 11:161-175. CrossRef Medline

Gardner SM, Takamiya K, Xia J, Suh JG, Johnson R, Yu S, Huganir RL (2005) Calcium-permeable AMPA receptor plasticity is mediated by subunitspecific interactions with PICK1 and NSF. Neuron 45:903-915. CrossRef Medline

Gauthier-Campbell C, Bredt DS, Murphy TH, El-Husseini Ael-D (2004) Regulation of dendritic branching and filopodia formation in hippocampal neurons by specific acylated protein motifs. Mol Biol Cell 15:22052217. CrossRef Medline

Greaves J, Prescott GR, Gorleku OA, Chamberlain LH (2009) The fat controller: roles of palmitoylation in intracellular protein trafficking and targeting to membrane microdomains (review). Mol Membr Biol 26:67-79. CrossRef Medline

Groc L, Choquet D (2006) AMPA and NMDA glutamate receptor trafficking: multiple roads for reaching and leaving the synapse. Cell Tissue Res 326:423-438. CrossRef Medline 
Jennings BC, Nadolski MJ, Ling Y, Baker MB, Harrison ML, Deschenes RJ, Linder ME (2009) 2-Bromopalmitate and 2-(2-hydroxy-5-nitrobenzylidene)-benzo[b]thiophen-3-one inhibit DHHC-mediated palmitoylation in vitro. J Lipid Res 50:233-242. CrossRef Medline

Jin W, Ge WP, Xu J, Cao M, Peng L, Yung W, Liao D, Duan S, Zhang M, Xia J (2006) Lipid binding regulates synaptic targeting of PICK1, AMPA receptor trafficking, and synaptic plasticity. J Neurosci 26:2380-2390. CrossRef Medline

Linden DJ, Connor JA (1991) Participation of postsynaptic PKC in cerebellar long-term depression in culture. Science 254:1656-1659. CrossRef Medline

Madsen KL, Beuming T, Niv MY, Chang CW, Dev KK, Weinstein H, Gether U (2005) Molecular determinants for the complex binding specificity of the PDZ domain in PICK1. J Biol Chem 280:20539-20548. CrossRef Medline

Mukai J, Liu H, Burt RA, Swor DE, Lai WS, Karayiorgou M, Gogos JA (2004) Evidence that the gene encoding ZDHHC8 contributes to the risk of schizophrenia. Nat Genet 36:725-731. CrossRef Medline

Mukai J, Dhilla A, Drew LJ, Stark KL, Cao L, MacDermott AB, Karayiorgou M, Gogos JA (2008) Palmitoylation-dependent neurodevelopmental deficits in a mouse model of 22q11 microdeletion. Nat Neurosci 11:13021310. CrossRef Medline

Nakamura Y, Wood CL, Patton AP, Jaafari N, Henley JM, Mellor JR, Hanley JG (2011) PICK1 inhibition of the Arp2/3 complex controls dendritic spine size and synaptic plasticity. EMBO J 30:719-730. CrossRef Medline

Ohno Y, Kihara A, Sano T, Igarashi Y (2006) Intracellular localization and tissue-specific distribution of human and yeast DHHC cysteine-rich domain-containing proteins. Biochim Biophys Acta 1761:474-483. CrossRef Medline
Rocca DL, Martin S, Jenkins EL, Hanley JG (2008) Inhibition of Arp2/3mediated actin polymerization by PICK1 regulates neuronal morphology and AMPA receptor endocytosis. Nat Cell Biol 10:259-271. CrossRef Medline

Sdrulla AD, Linden DJ (2007) Double dissociation between long-term depression and dendritic spine morphology in cerebellar Purkinje cells. Nat Neurosci 10:546-548. CrossRef Medline

Shepherd JD, Huganir RL (2007) The cell biology of synaptic plasticity: AMPA receptor trafficking. Annu Rev Cell Dev Biol 23:613-643. CrossRef Medline

Steinberg JP, Takamiya K, Shen Y, Xia J, Rubio ME, Yu S, Jin W, Thomas GM, Linden DJ, Huganir RL (2006) Targeted in vivo mutations of the AMPA receptor subunit GluR2 and its interacting protein PICK1 eliminate cerebellar long-term depression. Neuron 49:845-860. CrossRef Medline

Takamiya K, Mao L, Huganir RL, Linden DJ (2008) The glutamate receptor-interacting protein family of GluR2-binding proteins is required for long-term synaptic depression expression in cerebellar Purkinje cells. J Neurosci 28:5752-5755. CrossRef Medline

Thomas GM, Hayashi T, Chiu SL, Chen CM, Huganir RL (2012) Palmitoylation by DHHC5/8 targets GRIP1 to dendritic endosomes to regulate AMPA-R trafficking. Neuron 73:482-496. CrossRef Medline

Trinidad JC, Thalhammer A, Specht CG, Lynn AJ, Baker PR, Schoepfer R, Burlingame AL (2008) Quantitative analysis of synaptic phosphorylation and protein expression. Mol Cell Proteomics 7:684-696. CrossRef Medline

Zhou F, Xue Y, Yao X, Xu Y (2006) CSS-palm: palmitoylation site prediction with a clustering and scoring strategy (CSS). Bioinformatics 22:894896. CrossRef Medline 